\title{
SNAPSHOT OBSERVATIONS OF CIRCUMSTELLAR MATERIAL AROUND YOUNG STELLAR OBJECTS
}

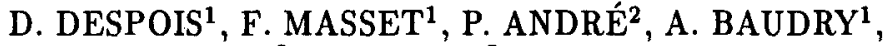 \\ T. MONTMERLE ${ }^{2}$, S. CABRIT ${ }^{3}$ \\ 1 Observatoire de Bordeaux, B.P.89, F-33270 Floirac \\ ${ }^{2}$ Service d'Astrophysique, C.E.A. Saclay, F-91191 Gif-sur-Yvette Cedex \\ ${ }^{3}$ Observatoire de Grenoble, B.P. 53X, F-38041 Grenoble Cedex
}

\begin{abstract}
We have undertaken $3 \mathrm{~mm}$ continuum and ${ }^{13} \mathrm{CO}$ line observations of 10 Young Stellar Objects with the Plateau de Bure Interferometer (PdBI) in a snapshot mode. We discuss here mainly the continuum dust emission (opacity index $\beta$ and source extent).
\end{abstract}

\section{INTRODUCTION}

The size, shape and gas to dust ratio of the circumstellar matter are fundamental characteristics of protostars and PMS stars. Millimetric interferometers are especially well suited to such studies as they trace both gas and dust with 2-3" resolution ( $\mathrm{a}$ few $100 \mathrm{AU}$ ). However only a limited number of objects have been mapped up to now (see the review by Sargent, these proceedings), and comparative studies have just begun (e.g. Ohashi et al., 1991 and these proceedings). To study a sample of 10 sources, we used the snapshot mode of PdBI : 1-2 h per object in each of the five 3-antennas configurations.

Our sample (Fig 1) includes 2 "class 0" candidates (André et al 1993), 5 classical T Tauri stars (cTTs), 1 Herbig AeBe, and two more luminous sources. There are 6 visible and 4 embedded objects ; 3 sources exhibit a bipolar outflow and one a monopolar outflow. There are indications from IR or radio observations that S68, L723 and Elias 1 are double. All objects were previously observed as strong sources at $1.3 \mathrm{~mm}(0.2-2 \mathrm{Jy})$.

\section{RESULTS AND DISCUSSION}

All sources but B335 were detected in the continuum in these snapshot observations, due to the excellent sensitivity of PdBI $(\sigma \sim 1.3-2 \mathrm{mJy} /$ beam $)$. One source, DL Tau, had already been observed with the NRO interferometer (Ohashi et al., 1991) ; both fluxes are in good agreement.

We have compared our $3 \mathrm{~mm}$ continuum fluxes to $1.3 \mathrm{~mm}$ data obtained with the MPI bolometer at IRAM 30m (Beckwith et al. 1990, Cabrit and André 1991, André et al. 1990 and in prep.). The derived ratio is plotted in Fig. 1. The inner error bar corresponds to 1 sigma of the estimated random noise on the ratio ; the outer error bar includes the calibration uncertainty.

The absorption coefficient of the dust is ill known at $\mathrm{mm}$ frequencies and is 


\begin{tabular}{|c|c|c|c|c|c|}
\hline SOURCE & \multicolumn{2}{|c|}{ type } & flow & $d(p c)$ & $L(L)$ \\
\hline S68 & B? & emb. & bip. & $>500$ & 300 \\
\hline LKHa234 & Herbig Be & vis. & mono. & 1000. & 130 \\
\hline Elias 24 & cTTs & emb. & - & 160. & 3.2 \\
\hline B335 & cl. 0 & emb. & bip. & 250 & 3 \\
\hline L723-mm & cl. 0 & emb. & bip. & 300 & 3 \\
\hline Elias 1 & Herbig Ae & vis. & $=$ & 140 & 0.7 \\
\hline GMAur & cTाs & vis. & $=$ & 140 & 0.7 \\
\hline DL Tau & cTTs & vis. & $=$ & 140 & 0.8 \\
\hline RY Tau & cits & vis. & $E$ & 140 & 7.5 \\
\hline CI Tau & cTT's & vis. & E & 140 & 1.1 \\
\hline
\end{tabular}

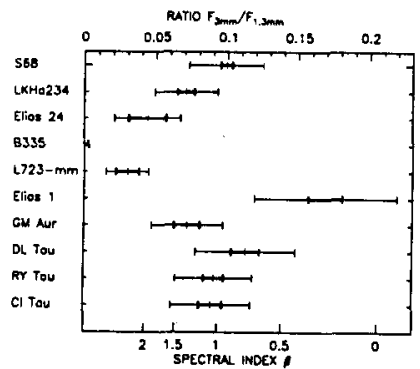

FIGURE I Table : Our source sample. Fig : Flux ratio $\left.F_{\nu(3 m m)} / F_{\nu(1.3 m m)}\right)$ and dust opacity index $\beta$

usually derived from IR values assuming a power-law in $\nu^{\beta}$. We show in Fig 1 (lower scale) the values derived in the Rayleigh-Jeans approximation, assuming the dust is optically thin and the source is pointlike.

For the 4 cTTs (GM Aur, RY Tau, CI Tau, DL Tau), which we consider essentially pointlike, $\beta$ is rather well determined, and in the range 1-1.5. This value is lower than that for the interstellar medium $(\beta=2)$, which is possibly due to grain aggregation. This is however higher than the values $\beta \sim-1$ to 1 derived by Beckwith and Sargent (1991) at higher frequencies $(\geq 230 \mathrm{GHz})$ and assuming optical thinness. Note that the two luminous sources S68 and LkH $\alpha 238$ appear to have $\beta$ in the same range as the cTTs.

Opacity effects may play a role. We considered values of $\tau_{1.3 \mathrm{~mm}}$ between 0 and 2 , uniform over the disc. This brings $\beta$ towards higher values but does not reconcile our data with the ISM value of 2 . We have checked also that the Rayleigh-Jeans approximation does not affect much the derived value for $\beta$ at the expected dust temperatures $(\sim 20-50 \mathrm{~K})$.

The low $\left(F_{\nu(3 \mathrm{~mm})} / F_{\nu(1.3 \mathrm{~mm})}\right)$ flux ratio derived for the two most embedded sources (B335, L723-mm) is best explained by flux missed by the interferometer because of non detected extended structure. A full synthesis of these objects with better uv coverage is needed to check this idea.

The flux ratio for Elias 1 is especially high. This may indicate special dust properties (this source exhibits IR absorption lines of $\mathrm{H} 2 \mathrm{O}$ and $\mathrm{CO}$ ices.), or free-free emission as in $\mathrm{T}$ Tau.

Several sources were detected in ${ }^{13} \mathrm{CO}$, including the embedded source B335 not detected in continuum. This latter source shows an elongated structure at $V_{l s r} \approx 7-8.7 \mathrm{~km} / \mathrm{s}$, whose N-W to S-E direction is roughly tangent to the outflow.

\section{REFERENCES}

André, P., Montmerle T., Feigelson, E.D., Steppe, H. 1990, A\&A, 240, 321 André, P., Ward-Thomson D., Barsony, M. $1993 A p J$, accepted (March 20) Beckwith, S.V.W., Sargent, A.I. 1991, ApJ, 381, 250

Beckwith, S.V.W., Sargent, A.I., Chini, R.S., Güsten, R. 1990, $A J, 99,924$

Cabrit S., André P. 1991, ApJ, 379, L25

Ohashi, N., Kawabe, R., Hayashi, M., Ishiguro, M. 1991, $A J, 102,2054$ 